\title{
Randomized Comparison of 3 Methods to Screen for Domestic Violence in Family Practice
}

Ping-Hsin Chen, PbD

Sue Rovi, $P b D$

Judy Washington, MD

Abbie Jacobs, MD

Marielos Vega, BSN, RN

Ko-Yu Pan, PbD

Mark S. Jobnson, MD, MPH

Department of Family Medicine, New Jersey Medical School, University of Medicine and Dentistry of New Jersey, Newark, NJ

Conflicts of interest: none reported

\section{CORRESPONDING AUTHOR}

Ping-Hsin Chen, PhD

Department of Family Medicine, UMDNJ - NJMS

185 S. Orange Ave

Newark, NJ 07103

chenpi@umdnj.edu

\begin{abstract}
PURPOSE We undertook a study to compare 3 ways of administering brief domestic violence screening questionnaires: self-administered questionnaire, medical staff interview, and physician interview.

METHODS We conducted a randomized trial of 3 screening protocols for domestic violence in 4 urban family medicine practices with mostly minority patients. We randomly assigned 523 female patients, aged 18 years or older and currently involved with a partner, to 1 of 3 screening protocols. Each included 2 brief screening tools: HITS and WAST-Short. Outcome measures were domestic violence disclosure, patient and clinician comfort with the screening, and time spent screening.
\end{abstract}

RESULTS Overall prevalence of domestic violence was 14\%. Most patients (93.4\%) and clinicians (84.5\%) were comfortable with the screening questions and method of administering them. Average time spent screening was 4.4 minutes. Disclosure rates, patient and clinician comfort with screening, and time spent screening were similar among the 3 protocols. In addition, WAST-Short was validated in this sample of minority women by comparison with HITS and with the 8-item WAST.

CONCLUSIONS Domestic violence is common, and we found that most patients and clinicians are comfortable with domestic violence screening in urban family medicine settings. Patient self-administered domestic violence screening is as effective as clinician interview in terms of disclosure, comfort, and time spent screening.

Ann Fam Med 2007;5:430-435. DOI: 10.1370/afm.716.

\section{INTRODUCTION}

$\mathrm{T}$

The prevalence of current victims of domestic violence among patients in primary care settings ranges from $7 \%$ to $50 \%,{ }^{1}$ even though studies show that only $1 \%$ to $15 \%$ of women in primary care settings were asked about domestic violence by their clinician. ${ }^{2-4}$ Lack of office protocols and limited time are perceived as common barriers by medical clinicians. $^{5-8}$ In one study, battered women perceived clinician reluctance to ask about abuse as a major barrier to their domestic violence disclosure. ${ }^{9}$

Although studies have found that brief screening questionnaires increase identification of domestic violence, ${ }^{10,11}$ research findings are inconsistent on the optimum method of administering screening tests. In a recent randomized study, MacMillan et al found no significant difference in the proportion of patients who disclosed domestic violence using a selfadministered questionnaire compared with patients who were interviewed by a clinician, the patients, however, preferred self-administered screening. ${ }^{12}$ McFarlane and colleagues found that a medical staff interview identified more abused women than a written history form ${ }_{1}^{13}$ whereas another study reported opposite findings. ${ }^{14} \mathrm{~W}$ ith few notable exceptions, ${ }^{10,12-17}$ 
previous studies have not examined clinician and patient comfort with different screening protocols. One study of a brief screening tool indicated that $91 \%$ of women felt comfortable when screened by their clinicians. ${ }^{10}$

The purpose of this study was to identify an optimal screening protocol to help overcome barriers to domestic violence screening. We compared the rate of domestic violence disclosure, comfort level with screening, and time spent screening for self-administered, medical staff interview, and physician interview screening protocols.

\section{METHODS \\ Participants}

This randomized trial of 3 domestic violence screening protocols was conducted in 4 urban family medicine practices. The 4 practices have a total of 18 physicians and 27 residents who see approximately 3,500 patients per month total, of which $86 \%$ are African-American or Hispanic. The target population was women aged 18 years or older who were currently with a partner.

\section{Procedure}

Figure 1 is a flowchart of the study design. At the start of the study, 7 research assistants, all medical staff, and all physicians received 3 hours of training by the investigators in lecture format on screening techniques, the use of the screening questionnaires, assessment of domestic violence victims, interventions, and referral to support agencies.

During June 2004 through March 2005, the research assistants approached and recruited consecutive patients and confirmed their eligibility in private. Screening at each practice site ranged from 2 to 5 months, because some practice sites see more adult female patients and took fewer months to accrue the sample. The research assistants completed the study in one practice before moving on to the next. Randomization of the 3 protocols was conducted before recruitment with research assistants blinded to the method assigned to patients. A block design was not used. If the protocol was to be self-administered, the patient completed the
Figure 1. Flowchart of study design.

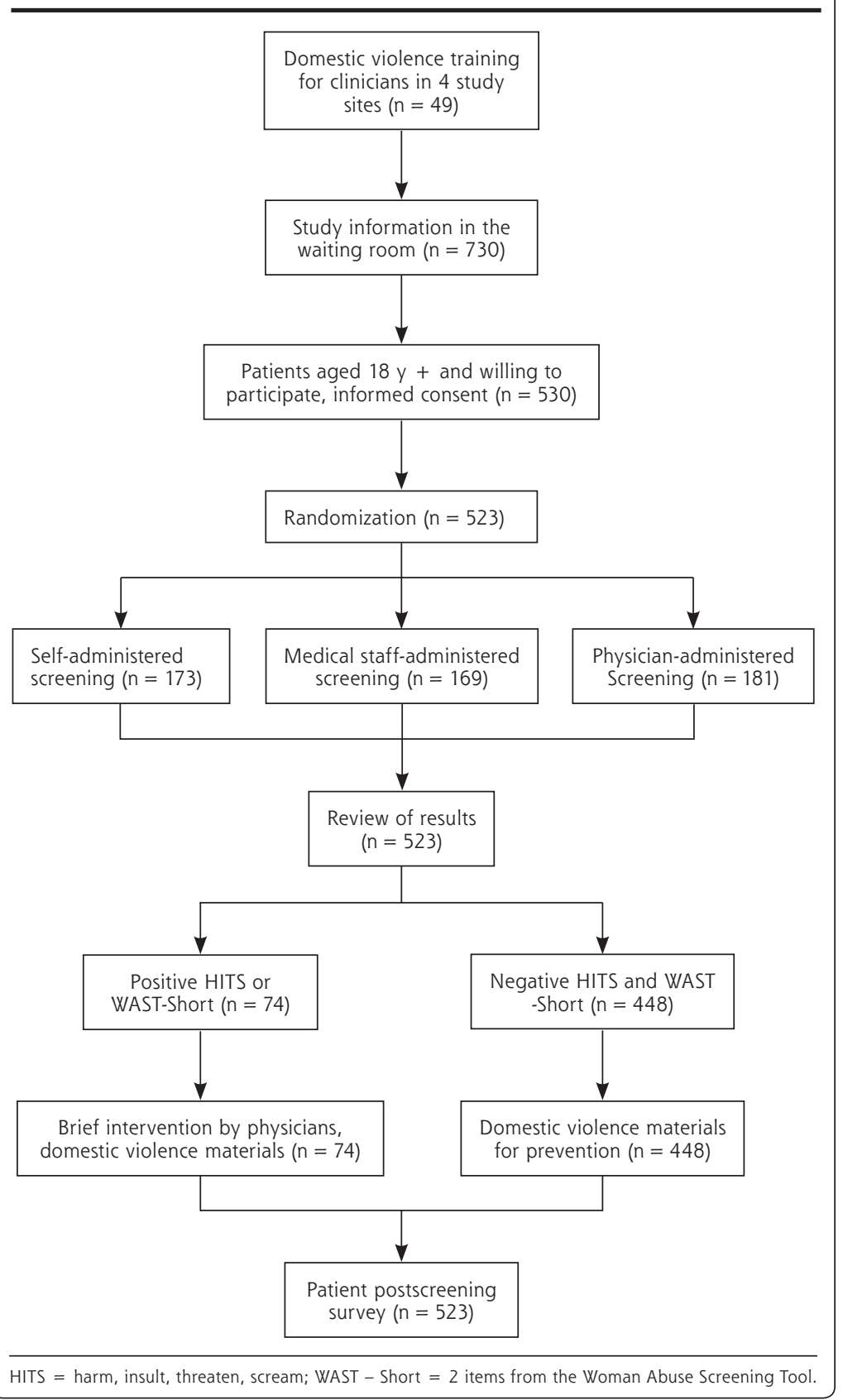


ing test was positive for domestic violence received an intervention by the physicians. The time taken to provide materials on domestic violence was included in the estimate of time for screening, whereas the time taken to intervene with those who disclosed domestic violence was not included.

After the health care visit, the research assistants distributed self-administered questionnaires to the participants in a private room. This postscreening questionnaire sought more details about the domestic violence and assessed comfort level and time spent screening. After patients completed the questionnaire, the women received a stipend of $\$ 10$ for their participation. At the end of the study, medical staff and physicians completed a self-administered questionnaire on their time spent screening and their overall comfort with screening but not about a specific patient.

\section{Data Collection}

Patients were screened for domestic violence using 2 brief screening tools: HITS (hurt-insult-threatenscream) ${ }^{18}$ and WAST (Woman Abuse Screening Tool)-Short ${ }^{16}$ (Supplemental Appendix at: http://www.

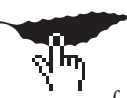

annfammed.org/cgi/content/full/5/5/430/DC1).

HITS is one of the shortest screening tools, forms an easily remembered acronym, has been tested with diverse populations, and has been tested and used in family medicine practices. Answers were summed to form an interval scale of the total HITS score, which could range from 4 to 20. Using a cutoff score of 10.5 , Sherin et al found that HITS accurately classified $91 \%$ of nonvictims and $96 \%$ of victims. ${ }^{18}$ HITS has a Cronbach's $\alpha$ of 0.80 and is highly correlated with the Conflict Tactics Scales $(r=0.85) .{ }^{18,19}$

WAST-Short consists of 2 items from the 8 -item WAST. Women responded to the 2 items with a 3 point response set and met the criteria for domestic violence exposure if they answered "a lot of tension/ great difficulty" to either question. The remaining 6 items were included in the postscreening questionnaire to validate results from the domestic violence screening. For the 8 -item WAST, answers were summed to form an interval scale, ranging from 3 to 24 . The WAST has a reliability of 0.75 , and abused women identified by WAST-Short score significantly higher on WAST than women who were not abused. ${ }^{10}$ WAST has been tested in only predominantly white and middleclass populations, however. ${ }^{10}$

Women who had positive findings on HITS or WAST-Short in the 3 protocols met the criteria for domestic violence exposure.

Data on patient comfort levels with the screening instruments, the screening method, and time spent screening were collected using a patient postscreen- ing questionnaire. Questions addressing comfort with screening were adapted from previous studies, ${ }^{10,16}$ with answers ranging from not at all (1) to very comfortable (4). Patient comfort levels with each item of HITS and WAST were assessed in the postscreening questionnaire (Supplemental Appendix). Answers were summed to form interval scales of the total HITS, WAST, and overall comfort scores. We included a question on how comfortable the patient was with the assigned screening method. A clinician survey assessed the physicians' and medical staff's comfort level with the screening tools and the assigned screening protocol. Scales similar to the patient comfort scales were used.

We calculated the sample size based on a previous study by the authors and the literature. ${ }^{10,13,19} \mathrm{We}$ expected patient disclosure rates of $6 \%$ for the selfadministered questionnaire, $16 \%$ for the medical staff interview, and $9 \%$ for the physician interview. We hypothesized 0.3 differences in comfort level scores $(3.0,3.3,3.6$, respectively; $\mathrm{SD}=0.5)$. Screening for domestic violence with 167 women from each method for a total of 501 provides more than $80 \%$ power to detect such differences at $\alpha=.05$ (2-tailed test). Institutional Review Board approval for this study was obtained.

\section{Analysis}

Analysis was done using SPSS version 14.0.2 (SPSS, Inc, Chicago, Ill). Outcome measures were domestic violence disclosure, patient or clinician comfort level, and time spent screening. To determine the reliability and validity of HITS and WAST-Short, we calculated Cronbach's coefficient $\alpha$, correlations of the 2 instruments with WAST, and assessed the relation of domestic violence disclosure by HITS and WAST-Short to WAST scores. Then, $\chi^{2}$ tests and ANOVA were conducted to compare differences in domestic violence disclosure, patient and clinician comfort levels, and time spent screening among the 3 protocols.

\section{RESULTS \\ Participants}

During the 10 months in the practices, 730 women were eligible to participate in the study. Of these women, 200 refused to participate, and $7 \mathrm{did}$ not complete the questionnaire because of the waiting time for a private room. A total of 523 (72\%) eligible patients participated in the study. Institutional Review Board regulations did not allow us to collect demographic data for nonrespondents; however, percentages of African American and Hispanic women in the sample and the 4 practices were similar ( $84 \%$ and $86 \%$, respectively). 


\begin{tabular}{|c|c|c|c|c|c|}
\hline Variable & $\begin{array}{c}\text { Total } \\
n=523\end{array}$ & $\begin{array}{c}\text { Self-Report } \\
n=173\end{array}$ & $\begin{array}{c}\text { Medical Staff } \\
\text { Interview } \\
n=169\end{array}$ & $\begin{array}{c}\text { Physician } \\
\text { Interview } \\
n=181\end{array}$ & $P$ Value \\
\hline \multicolumn{6}{|l|}{ Race/ethnicity, \% } \\
\hline White & 11.7 & 15.1 & 10.7 & 9.4 & .769 \\
\hline African American & 70.6 & 67.4 & 70.8 & 73.3 & \\
\hline Hispanic & 13.8 & 13.4 & 14.9 & 13.3 & \\
\hline Other & 3.8 & 4.1 & 3.6 & 3.9 & \\
\hline Age, mean, y & 35.6 & 35.8 & 35.9 & 35.3 & .886 \\
\hline Completed college, \% & 29.5 & 32.4 & 29.0 & 27.2 & .561 \\
\hline Income, mean, \$ & 20,423 & 20,232 & 20,650 & 20,388 & .974 \\
\hline Employed (including part-time), \% & 72.6 & 64.5 & 80.4 & 72.9 & .005 \\
\hline Married, \% & 33.4 & 28.3 & 38.9 & 33.1 & .117 \\
\hline Currently pregnant, $\%$ & 4.5 & 4.1 & 4.8 & 4.5 & .953 \\
\hline Length of the relationship, mean, $y$ & 8.2 & 7.8 & 8.9 & 7.9 & .586 \\
\hline Medicare/Medicaid, \% & 51.5 & 57.6 & 47.3 & 49.7 & .140 \\
\hline New patient, \% & 19.8 & 16.8 & 24.6 & 18.4 & .167 \\
\hline Visits in the past year, mean, No. & 4.3 & 4.8 & 4.2 & 3.8 & .005 \\
\hline
\end{tabular}

Table 1 displays the demographic characteristics of participants. The 3 randomly allocated groups were similar on all but 2 variables. Women interviewed by medical staff were more likely to be employed $(P=.005)$, and those who completed self-administered questionnaires had visited the practice more often in the previous year $(P=.005)$.

\section{Disclosure of Domestic Violence}

Table 2 shows no difference in the prevalence of a positive screening result for domestic violence among 3 methods of administering HITS and WAST-Short. Overall, $14.2 \%$ of women had positive findings on 1 or both instruments. A higher proportion of women had positive findings with WAST-Short than with HITS $(12.5 \%$ vs $6.3 \%$, respectively), perhaps because the WAST-Short questions are more general.

\section{Internal Reliability and Validity of Instruments}

The internal reliability of the short screening instruments was good, and each was highly correlated with the WAST total score. Cronbach's $\alpha$ was .79 for HITS and .80 for WAST. The correlations of HITS and WASTShort total scores with WAST total scores were 0.77 $(P<.001)$ and $0.81(P<.001)$, respectively. Those who had positive results on HITS or WAST-Short had WAST total scores that were significantly higher than those who had negative results ( $P<.001$ for both instruments). Cronbach's $\alpha$ was .97 for the patient overall comfort scale and .95 for the clinician overall comfort scale.

\section{Patient Comfort and Time Spent Screening}

Most patients $(93.4 \%$; total $\mathrm{n}=523)$ reported being comfortable with the assigned protocol. Comparisons of the mean scores of patient comfort showed no differences in HITS, WAST-Short, and overall comfort among protocols. Women whose screening results were positive for domestic violence and those whose were not were similar in perception of helpfulness of screening and comfort with screening across methods. One exception was that those with positive screening results for domestic violence were slightly less comfortable with medical staff screening $(P=.021)$. Time spent screening ranged from 2 to 15 minutes and was similar among the 3 methods.

\section{Clinician Comfort}

Most clinicians $(84.5 \%$; total $n=33)$ were comfortable with the screening methods regardless of whether the clinician interviewed the patient or the questionnaire was self-administered. Comfort levels with each screening protocol were similar for physicians and medical staff across methods. Physicians and medical staff were comfortable with the screening tools (mean score $=3.1$ ), although physicians were slightly more comfortable with WAST-Short compared with the medical staff $(P=.039)$. Subgroup analysis indicated that physicians preferred WAST-Short to HITS $(P=.043)$, and self-administered questionnaire to medical staff interview $(P=.007)$.

\section{DISCUSSION}

Our findings provide support that rates of domestic violence disclosure are similar with self-administered screening, medical staff interview, and physician interview. The prevalence rate of domestic violence in our study is similar to findings from previous studies. ${ }^{12-14}$ 
Table 2. Patient Disclosure and Patient and Clinician Comfort Level, by Screening Protocol

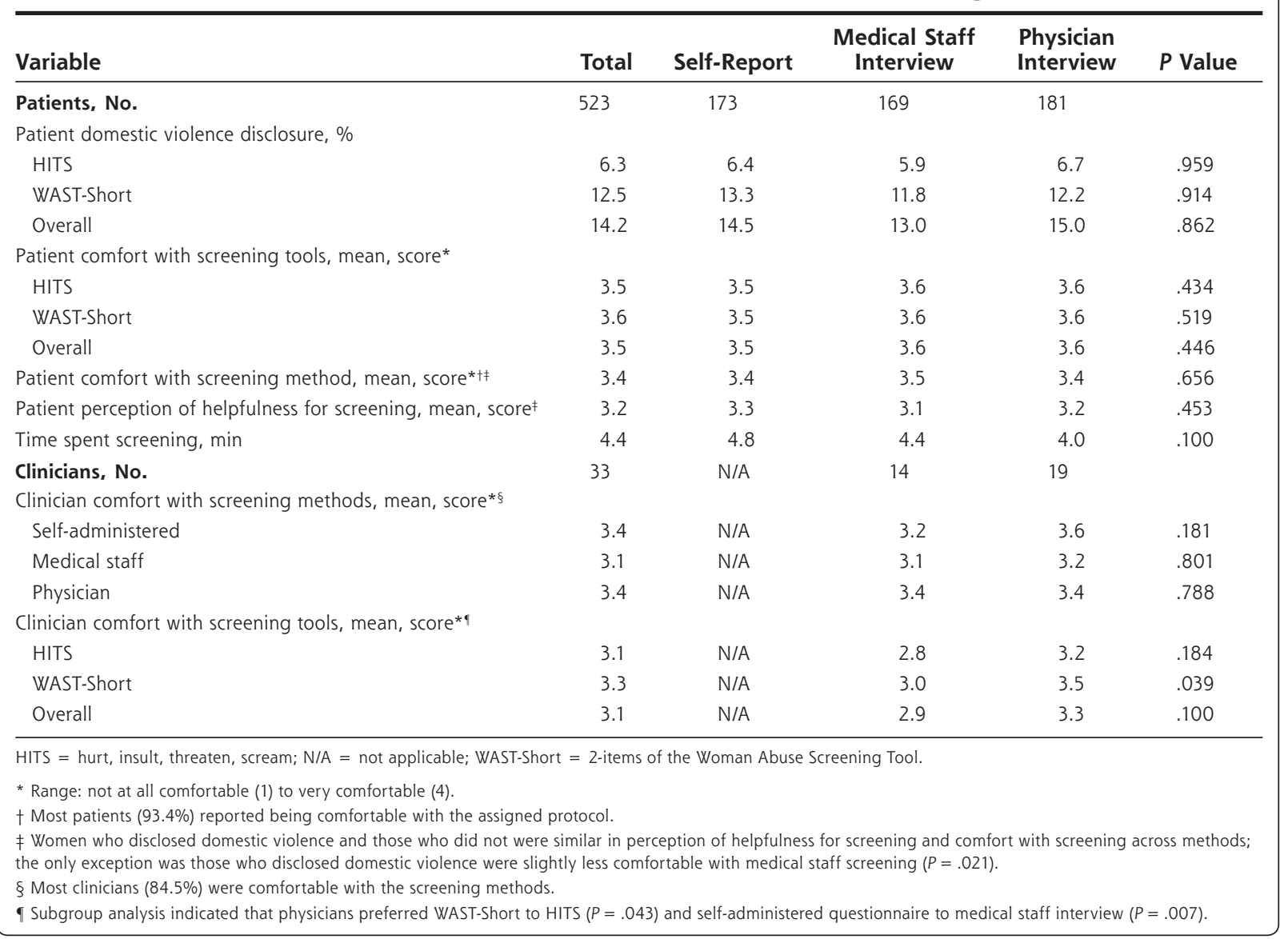

Overall, 1 in 7 women in intimate relationships had positive findings for domestic violence. Consistent with prior studies, our findings show that patients and clinicians are comfortable with domestic violence screening. ${ }^{10,13-17}$ Although MacMillan et al found that a face-to-face approach was less preferred by patients, ${ }^{12}$ we found that patient comfort levels were similar among clinician interviews and self-completed questionnaires. We also found that physicians were comfortable regardless of screening tools and screening methods. This study provides implications for cost savings if self-administered screening tools are used.

This study has some limitations. Provision of a stipend might affect the participant's rating of their comfort with screening. Even so, the stipend did not seem to affect the comparative ratings of the 3 screening protocols. There was no validation of the time spent , however, the reported time is reasonable given the brevity of the questionnaire. Nonparticipants and participants might differ in demographic characteristics and in abuse, which may introduce unknown bias. Our study only screened women patients, because women are 7 to 14 times more likely than men to suffer injuries. ${ }^{20}$ We did not have physicians and medical staff assess their comfort with screening each patient. Finally, this study was conducted in urban clinical settings with predominantly minority patient populations, indicating that WAST and WAST-Short may be acceptable screening instruments in such settings.

In conclusion, domestic violence is common in urban family medicine settings. Screening patients for domestic violence using a self-administered questionnaire is as effective as clinician screening in terms of disclosure, comfort, and time spent screening. Brief screening tools such as those used in this study can be helpful to busy clinicians, but in practice, clinicians would still need to conduct further assessment to confirm the domestic violence victim's status.

To read or post commentaries in response to this article, see it online at http://www.annfammed.org/cgi/current/full/5/5/430.

Key words: Domestic violence/diagnosis; screening

Submitted July 3, 2006; submitted revised March 7, 2007; accepted March 26, 2007. 
An earlier version of this article was presented at the North American Primary Care Research Group (NAPCRG) 33th Annual Meeting, October 2005, Quebec City, Quebec.

Funding support: Preparation of this article was supported by a grant from the American Academy of Family Physicians Foundation (\#G0415).

Acknowledgments: The authors thank the departmental writing group for their comments on an earlier version of this manuscript.

\section{References}

1. Coker AL, Reeder CE, Fadden MK, Smith PH. Physical partner violence and Medicaid utilization and expenditures. Public Health Rep. 2004;119(6):557-567.

2. Naumann P, Langford D, Torres S, Campbell J, Glass N. Women battering in primary care practice. Fam Pract. 1999;16(4):343-352

3. Friedman LS, Samet JH, Roberts MS, Hudlin M, Hans P. Inquiry about victimization experiences. A survey of patient preferences and physician practices. Arch Intern Med. 1992;152(6):1186-1190.

4. Thompson RS, Rivara FP, Thompson DC, et al. Identification and management of domestic violence: a randomized trial. Am J Prev Med. 2000;19(4):253-263.

5. Erickson MJ, Hill TD, Siegel RM. Barriers to domestic violence screening in the pediatric setting. Pediatrics. 2001;108(1):98-102.

6. Cummins A, Little D, Seagrave M, Ricken A, Esparza V, RichardsonNassif K (2002). Vermont Family Practitioners' Perceptions on Intimate Partner Violence. North American Primary Care Research Group (NAPCRG) 30th Annual Meeting, New Orleans, LA.

7. Gerber MR, Leiter KS, Hermann RC, Bor DH. How and why community hospital clinicians document a positive screen for intimate partner violence: a cross-sectional study. BMC Fam Pract. 2005;6:48.

8. McGrath ME, Bettacchi A, Duffy SJ, Peipert JF, Becker BM, St Angelo L. Violence against women: provider barriers to intervention in emergency departments. Acad Emerg Med. 1997;4(4):297-300.
9. Rodriguez MA, Quiroga SS, Bauer HM. Breaking the silence. Battered women's perspectives on medical care. Arch Fam Med. 1996;5(3):153-158.

10. Brown JB, Lent B, Schmidt G, Sas G. Application of the Woman Abuse Screening Tool (WAST) and WAST-Short in the family practice setting. J Fam Pract. 2000;49(10):896-903.

11. Ramsay J, Richardson J, Carter YH, Davidson LL, Feder G. Should health professionals screen women for domestic violence? Systematic review. BMJ. 2002;325(7359):314.

12. MacMillan $\mathrm{HL}$, Wathen $\mathrm{CN}$, Jamieson $\mathrm{E}$, et al. Approaches to screening for intimate partner violence in health care settings: a randomized trial. JAMA. 2006;296(5):530-536.

13. McFarlane J, Christoffel K, Bateman L, Miller V, Bullock L. Assessing for abuse: self-report versus nurse interview. Public Health Nurs. 1991;8(4):245-250.

14. Canterino JC, VanHorn LG, Harrigan JT, Ananth CV, Vintzileos AM. Domestic abuse in pregnancy: A comparison of a self-completed domestic abuse questionnaire with a directed interview. Am J Obstet Gynecol. 1999;181(5 Pt 1):1049-1051.

15. Fogarty CT, Brown JB. Screening for abuse in Spanish-speaking women. J Am Board Fam Pract. 2002;15(2):101-111.

16. Brown JB, Lent B, Brett PJ, Sas G, Pederson LL. Development of the Woman Abuse Screening Tool for use in family practice. Fam Med. 1996;28(6):422-428.

17. Gerbert B, Bronstone A, Pantilat S, McPhee S, Allerton M, Moe J. When asked, patients tell: disclosure of sensitive health-risk behaviors. Med Care. 1999;37(1):104-111.

18. Sherin KM, Sinacore JM, Li XQ, Zitter RE, Shakil A. HITS: a short domestic violence screening tool for use in a family practice setting. Fam Med. 1998;30(7):508-512.

19. Chen PH, Rovi S, Vega M, Jacobs A, Johnson MS. Screening for domestic violence in a predominantly Hispanic clinical setting. Fam Pract. 2005;22(6):617-623.

20. Screening for family and intimate partner violence: recommendation statement. Ann Intern Med. 2004;140(5):382-386 